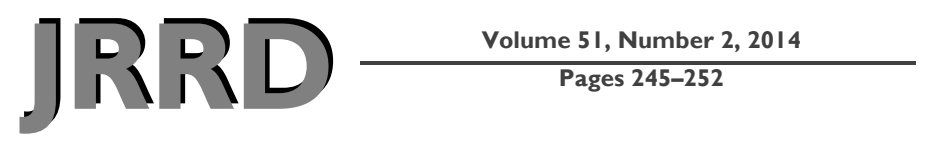

\title{
Complex muscle vibration patterns to induce gait-like lower-limb movements: Proof of concept
}

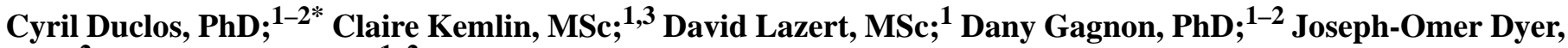 \\ PhD; ${ }^{2}$ Robert Forget, PhD $^{1-2}$ \\ ${ }^{1}$ Centre for Interdisciplinary Research in Rehabilitation, Institut de Réadaptation Gingras-Lindsay-de-Montréal, Mon- \\ tréal, Canada; ${ }^{2}$ School of Rehabilitation, Université de Montréal, Canada; ${ }^{3}$ Service de Médecine Physique et Réadap- \\ tation, Pitié Salpétrière, Paris, France
}

\begin{abstract}
Muscle vibrations can induce motor responses and illusions of complex movements. However, inducing gait-like cyclical movements and illusions requires the application of multiple fast alternating vibrations to lower-limb muscles. The objectives were (1) to test the feasibility of delivering complex vibrations in a time-organized manner and (2) to illustrate the possibility of inducing alternate gait-in-place-like movements using these vibrations. Patterns of vibration, produced by 12 vibrators applied bilaterally on the flexor and extensor muscle groups of the lower limbs, were based on normal gait kinematics. We tested $1 \mathrm{~s}$ and $2 \mathrm{~s}$ cycle patterns of vibration. Vibrator responses were assessed using auto- and crosscorrelations and frequency analyses based on accelerometry measurements, and compared between patterns. High auto- $(>0.8)$ and crosscorrelation (>0.6) coefficients demonstrated a good response by the vibrators to the control signal. Vibrations induced cyclical, low-amplitude stepping-in-place movements that mimicked alternate walking movements with both legs, with $1 \mathrm{~s}$ and $2 \mathrm{~s}$ cycle durations, in one nondisabled participant and one participant with American Spinal Injury Association Impairment Scale B spinal cord injury standing, relaxed, with body-weight support. Electromechanical vibrators can deliver complex cyclical vibrations and trigger gait-like lower-limb movements. These results warrant the application of these vibration patterns on individuals with sensorimotor impairments to test their potential in gait rehabilitation.
\end{abstract}

Key words: accelerometry, electromechanical vibrators, gait rehabilitation, humans, kinematics, locomotion, movement illusion, muscle vibration, neuromuscular stimulation, proprioception, spinal cord injury.

\section{INTRODUCTION}

Muscle vibration is a powerful somatosensory stimulation [1-3]. Vibration increases the discharge rate of muscle afferents, particularly the Ia afferents, to a frequency up to $100 \mathrm{~Hz}$ [2]. Because these afferents detect muscle lengthening, vibration induces a perception of an illusory lengthening movement of the vibrated muscle $[2,4]$. Vibration can also generate activity in the vibrated muscle or its antagonist [5-8]. Applied on the tibialis anterior at the ankles during standing, vibration also induces postural reactions. The amplitude of these reactions progressively increases with vibration frequency up to $100 \mathrm{~Hz}$ [9].

\footnotetext{
Abbreviations: FFT $=$ Fast Fourier Transform, $\mathrm{L}=$ lumbar.

*Address all correspondence to Cyril Duclos, PhD; Centre de Recherche Interdisciplinaire en Réadaptation (Université de Montréal), Institut de Réadaptation Gingras-Lindsayde-Montréal, 6300 avenue Darlington, Montréal, QC, H3S 2J4, Canada; 514-340-2111 ext 3048.

Email: cyril.duclos@umontreal.ca http://dx.doi.org/10.1682/JRRD.2013.04.0079
} 
Illusions of complex movements, such as writing, are also described when vibrations are applied in a proper sequence on several muscles [10]. Since muscle vibration can also induce motor activity associated with the illusion [7-8], multiple patterned vibrations applied on lower-limb muscles may trigger complex, organized movements similar to gait. However, because gait is cyclical, regular and fast alternating periods of vibration would have to be delivered with proper timing and frequency to obtain a gait-like illusion resulting in motor activity and movements.

We thus conducted the proof of concept of delivering complex vibrations in a time-organized manner and demonstrated the feasibility of inducing small amplitude, gait-like movements in a nondisabled participant and a participant with spinal cord injury using multiple vibrations.

\section{METHODS}

\section{Equipment}

Twelve vibrators (VB115, TECHNO CONCEPT; Mane, France) were bilaterally placed on the tendons of each flexor and extensor muscle group at the hip, knee, and ankle (Figure 1). The vibrators were controlled using a custom-made LabVIEW interface (National Instruments; Austin, Texas) and held in place using tubular jersey.

Two accelerometers (Analog Devices Inc; Norwood, Massachusetts) were fixed on pairs of vibrators to monitor their activation at a sampling frequency of $600 \mathrm{~Hz}$. During successive trials, the accelerations of each vibrator were simultaneously recorded with a reference vibrator placed over the right hip flexor muscles. Such a reference was necessary because only two accelerometers were available for data collection. It provided a temporal reference to compare the timing of the stimulations between the 12 vibrators.

\section{Tested Patterns}

Due to vibrator limitations associated with fast frequency changes observed in pretests, only one period of vibration could be delivered by each vibrator within each cycle of stimulation. The onset and duration of each period of vibration was based on normal gait kinematics [11] to activate somatosensory afferents according to muscle stretches associated with joint movements during

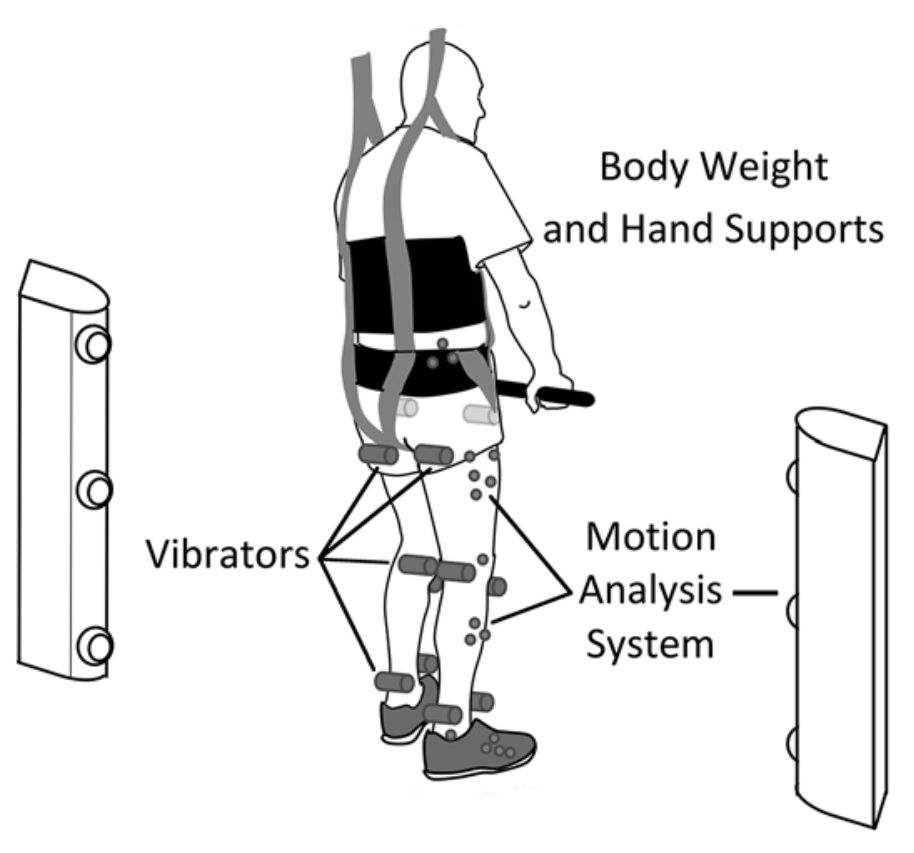

Figure 1.

Experimental setup: participants were equipped with body weight and hand supports, motion analysis system (34 infrared markers and 2 cameras) and 12 vibrators, one on tendon of each flexor and extensor muscle of hips, knees, and ankles.

gait (Figure 2(a)). The pattern was repeated to ensure cyclic stimulation over 1 min trials.

Patterns reproducing gait cycles of 1 and $2 \mathrm{~s}$ were tested, resulting in 60 or 30 consecutive repetitions of the model pattern of vibrations (Figure 2(a)) over the minute of stimulation, respectively. Patterns were also tested with symmetrical (bilateral target frequency of $80 \mathrm{~Hz}$ ) and asymmetrical ( $80 \mathrm{~Hz}$ on one leg, $115 \mathrm{~Hz}$ on the other leg) frequencies with identical timing. Because higher vibration frequency induces larger motor responses [9], asymmetrical stimulation, with the highest frequency applied to the most affected lower limb, could potentially increase the response on the affected side. Such asymmetrical vibration could be used in an attempt to obtain more symmetrical movements in individuals where asymmetrical deficits can be observed, such as following a stroke or spinal cord injury.

The symmetrical patterns were applied on one nondisabled participant and one participant with incomplete spinal cord injury during quiet standing. The clinical characteristics of the person with spinal cord injury were the following: 52 yr old, American Spinal Injury Association Impairment Scale B, at thoracic 12, 8.9 yr postinjury, 
(a) Model pattern (1 cycle on one limb)

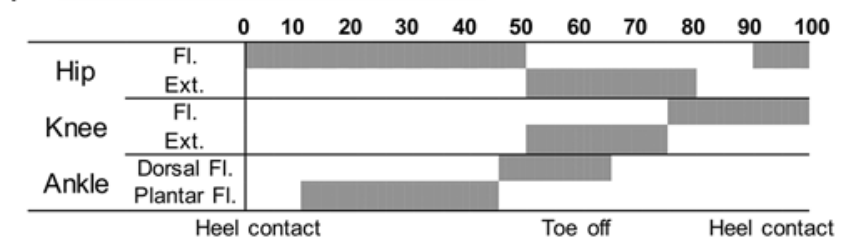

(b)

$\underline{1-\text { second pattern }}$

Hip vibration (Raw)

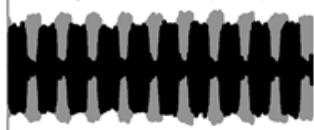

(c)
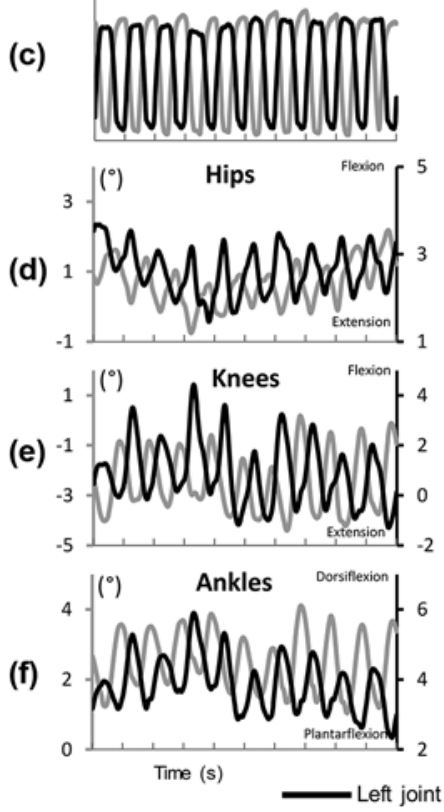

Hip vibration (RMS)

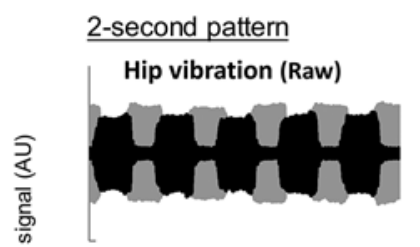

Hip vibration (RMS)
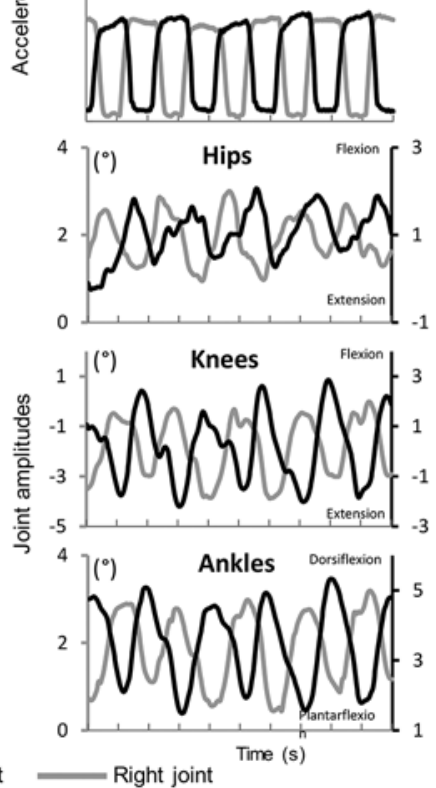

Figure 2.

(a) Schematic pattern of vibration, used as control signal to trigger complex vibration, based on gait kinematics (Ext = extensors, $\mathrm{Fl}=$ Flexors. Horizontal axis in \% of gait cycle). Vibration periods and joint amplitudes obtained in one nondisabled participant for $1 \mathrm{~s}$ (left) and $2 \mathrm{~s}$ (right) patterns. (b) Raw accelerometer signal recorded at hip flexors during vibration. (c) Root mean square (RMS)-enveloped accelerometer signal recorded at hip flexors during vibration, (d) hip, (e) knee, and (f) ankle joint angles (degrees); in gray (left vertical axis for (d),(e), and (f) for right lower limb and in black (right vertical axis for (d), (e), and (f) for left lower limb. AU = arbitrary units.

with good hip flexion and knee extension on the left side (4/5), but reduced strength on the right lower limb (2/5 for hip flexion and 1/5 for knee extension, for a total of
11/50 at the Lower Extremity Motor Score, and total bilateral paralysis in hip extension, knee flexion, and at the ankles). In terms of sensory deficits, touch was perceived as normal bilaterally at the lumbar (L)1 dermatome and at L2 and L3 on the left, but impaired at L2 and L3 on the right and absent below. Body-weight support was provided to reduce the need for reactions to postural destabilizations associated with lower-limb vibrations [9] and to facilitate a relaxed state. Both participants signed a consent form after complete information about the study was provided according to local ethics committee recommendations.

\section{Data Collection}

A Certus system (Northern Digital Inc; Waterloo, Canada) recorded the positions of at least three noncollinear infrared markers on each lower-limb segment (pelvis, thighs, legs and feet; see Figure 1 for details) at a sampling frequency of $60 \mathrm{~Hz}$ to compute a threedimensional link-segment model [12]. The joint amplitudes were calculated as the range of motion between the minimal and maximal angles of the hip, knee, and ankle joints as calculated according to the recommendations of the International Society of Biomechanics [13].

\section{Data Analysis}

Accelerometer signals were band-pass filtered between 10 and $150 \mathrm{~Hz}$. The peak frequency of the radial acceleration signal was determined using Fast Fourier Transforms (FFTs). The signal was then enveloped to obtain vibration profiles. In addition, to compare the timing of the stimulations between the 12 vibrators, the beginning of each vibration period was automatically determined from this profile to measure delays between each vibrator and the reference (right hip flexor vibrator).

Autocorrelation was computed to test the regularity [14] of the vibration periods on the time-series of the accelerometer-enveloped signal recorded for each vibrator over the minute of each trial. Crosscorrelations were used to compare each accelerometer-enveloped signal with its control signal [14] over the minute in each trial, with the control signal of each vibrator represented as rectangular pulses for each "on" period, with identical time parameters. We used $t$-tests on the correlation coefficients to determine whether the delivery of the vibrations was altered depending on the tested pattern. Mean and standard deviations of the duration and amplitude of the cyclical-induced joint movements, and their peak 
frequency determined by FFT, were calculated during the application of the vibration patterns. Statistics were computed using SPSS version 19 for Windows (IBM Corporation; Armonk, New York).

\section{RESULTS}

The mean frequency produced by the vibrators in the tested patterns was $88.3 \pm 5.1 \mathrm{~Hz}$ for the $80 \mathrm{~Hz}$ stimulations and $103.8 \pm 6.0 \mathrm{~Hz}$ for the $115 \mathrm{~Hz}$ stimulations.

Mean autocorrelation coefficients of the enveloped signals were very high for each vibrator but lower for the 1 s cycles than for the 2 s cycles $(p<0.05)$ (Table). Target frequencies did not affect the autocorrelation values $(p>0.20)$. The mean crosscorrelation between the control signals and the obtained vibrations for each pattern was lower for the $1 \mathrm{~s}$ patterns than for the $2 \mathrm{~s}$ symmetrical patterns $(p<0.05)$. In addition, the crosscorrelation was also lower in the asymmetrical patterns than in the symmetrical $1 \mathrm{~s}$ patterns $(p<0.01)$, but no difference appeared between the $2 \mathrm{~s}$ patterns $(p=0.64)$.

Compared with the delays set in the control signal, the mean error in the delays between the onset of the vibration periods of each vibrator and the reference vibrator was $22.4 \pm 22.9$ ms with no difference between conditions $(p>0.21)$.

Small amplitude (nondisabled participant: $2.3^{\circ} \pm 1.1^{\circ}$; spinal cord injured: $0.8^{\circ} \pm 0.3^{\circ}$ ) cyclical movements, with a 1.0 and $0.5 \mathrm{~Hz}$ peak frequency corresponding to the 1 and 2 s cycle duration of the vibration patterns, respectively, were recorded at each lower-limb joint during the stimulations. These movements alternated between the right and left lower limbs. In the nondisabled participant, hip flexion was accompanied by knee flexion and dorsiflexion on the same leg. This was followed by the reverse pattern of hip extension, knee extension, and plantar flexion (Figure 2). Movements differed in the participant with spinal cord injury (Figure 3), particularly at the hip, with flexion associated with knee extension and plantar flexion followed by extension associated with knee flexion and dorsiflexion on the same leg. The percentage of support used by each participant was 10 percent (nondisabled) and 88 percent (spinal cord injury) of total body weight.

\section{DISCUSSION}

Complex patterns of vibration were delivered by 12 electromechanical vibrators. The mean peak frequency was within the range known to activate somatosensory afferents at approximately $80 \mathrm{~Hz}$ [1-3]. There was a small frequency difference (approximately $20 \mathrm{~Hz}$ instead of the $35 \mathrm{~Hz}$ target) when using the asymmetrical pattern between the legs. The study was not designed to assess the reasons for the difference between the target vibration frequency and the actual vibration frequency. However, (a)

1-second pattern Hip vibration (Raw)

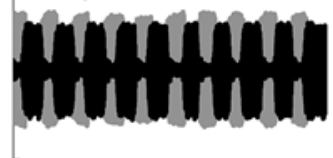

Hip vibration (RMS)

(b)

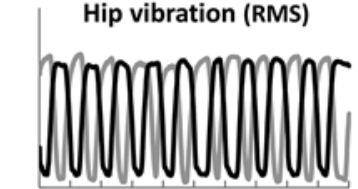

(c)

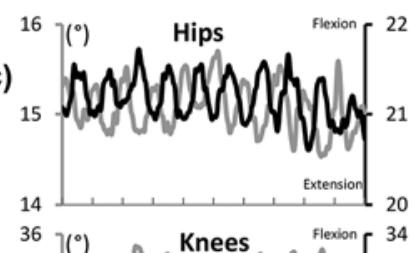

(d) 35
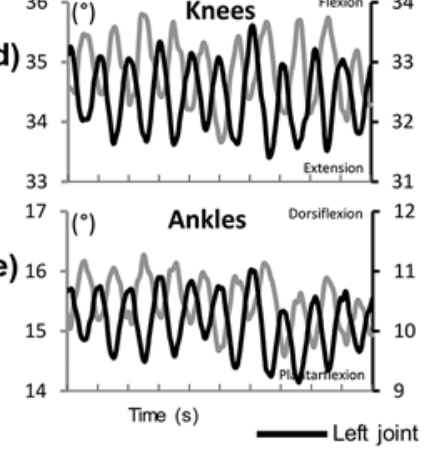

2-second pattern

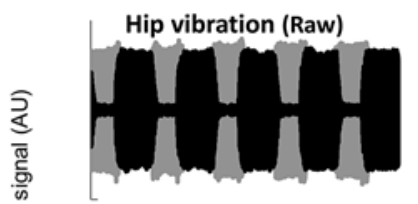

Hip vibration (RMS)
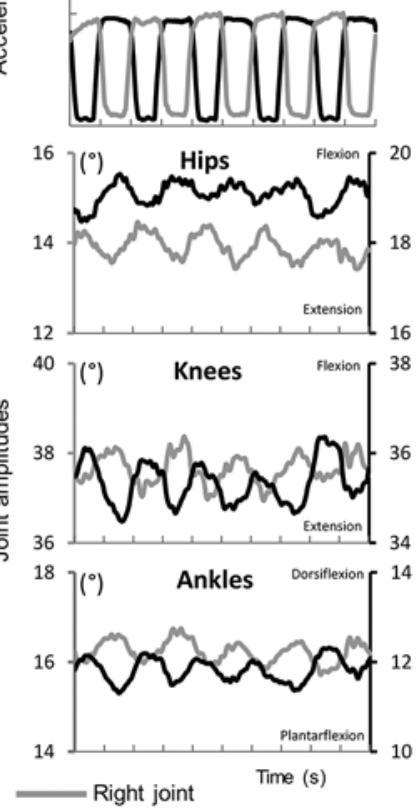

Figure 3.

Vibration periods and joint amplitudes obtained in one participant with American Spinal Cord Association Impairment Scale B (12th thoracic level) spinal cord injury for $1 \mathrm{~s}$ (left) and $2 \mathrm{~s}$ (right) patterns: (a) raw accelerometer signal recorded at hip flexors during vibration, (b) root mean square (RMS)-enveloped accelerometer signal recorded at hip flexors during vibration, (c) hip, (d) knee, and (e) ankle joint angles (in degrees); in gray (left vertical axis for (c), (d), and (e) for right lower limb) and in black (right vertical axis for (c), (d), and (e) for left lower limb). AU = arbitrary units. 
Table.

Regularity of vibration profile (mean autocorrelation coefficients) and its correspondence with control signal of tested pattern (mean crosscorrelation coefficients).

\begin{tabular}{|c|c|c|c|c|}
\hline Coefficient & $\begin{array}{c}1 \text { s, Symmetrical } \\
\text { Pattern }\end{array}$ & $\begin{array}{c}1 \text { s, Asymmetrical } \\
\text { Pattern }\end{array}$ & $\begin{array}{c}2 \text { s, Symmetrical } \\
\text { Pattern }\end{array}$ & $\begin{array}{c}2 \text { s, Asymmetrical } \\
\text { Pattern }\end{array}$ \\
\hline Autocorrelation & $0.89 \pm 0.03$ & $0.88 \pm 0.06$ & $0.95 \pm 0.01$ & $0.94 \pm 0.04$ \\
\hline
\end{tabular}

one can hypothesize that there could be flaws in the internal control of the vibrators (suboptimal control of frequency, limitation in power supply to the motors). Furthermore, the interface between the vibrators and the underlying skin/muscle tissue could influence the delivery of the vibration.

The responses of the vibrators to the control signal were good [14], with limited variations for each vibrator (autocorrelation above 0.8) and only slight mismatch between the control and vibration signals (lower crosscorrelation, particularly for the $1 \mathrm{~s}$ asymmetrical pattern). Lower crosscorrelation coefficients were likely due to the representation of the control signal as rectangular pulses, even though the frequency of the vibrators could not rise instantaneously. Lastly, the errors in the delays between vibrator responses were also limited, with mean errors of 25 ms. Thus, the vibrations produced by the 12 vibrators were very close to the control signal, the $2 \mathrm{~s}$ pattern being slightly better, particularly for the asymmetrical pattern. The development of vibrators with capacities to change their frequency faster and with more precise target frequency than those used in the present study would be necessary to apply vibration patterns closer to gait kinematics and at faster speed or to increase the stimulation at weaker joints for clinical applications. For example, Cordo et al. used isolated vibration at the ankle or wrist in persons with hemiparesis from stroke [15]. They assisted movement by applying vibration to the muscle that was stretched during alternate voluntary flexion and extension at the ankle or wrist. They used a two-phase vibration, with a higher frequency burst during the first 200 to $300 \mathrm{~ms}$ at $70 \mathrm{~Hz}$ followed by $60 \mathrm{~Hz}$ for the rest of the vibration period, to mimic the physiological response of muscle afferents to stretching. These authors attributed the measured functional improvements to better sensorimotor connections because of repetitive and simultaneous activation of sensory and motor neurons and cortices [15].

The complex vibration pattern generated alternating, low-amplitude, flexion-extension movements of both lower limbs at the frequency of the applied vibration periods. This finding provides good evidence to indicate that the applied vibrations were driving the generated movements. Unlike with air-stepping movements produced by the continuous vibration of one muscle group in the side lying position in relaxed nondisabled participants [16-17] and participants with spinal cord injury [18], it was thus possible to impose the frequency of the induced gait-like movements. This feature could be interesting for variations if used in gait training. In addition, one can be confident that the generated movements are actual movements since the motion analysis method used here has a bias of $0.05^{\circ}$ for angle measurement, well below the minimal amplitude measured in the present study, i.e., $0.8^{\circ} \pm$ $0.3^{\circ}[19]$.

Alternated movements were obtained both in the nondisabled participant and participant with spinal cord injury, though of lower amplitude for the latter. Characterizing the direct relationship between vibration patterns and muscle activity using electromyography was not possible given the complex electronic noise due to the vibrations and their variations. The difference observed between the two participants in the coordination of hip movements compared with knee and ankle movements may be due to the bilateral absence of hip extension and sensory perception at L4 to L5 in the participant with spinal cord injury. Further study in a larger group is needed to analyze the effect of the level of sensorimotor impairment and of the percentage of supported body weight on the vibration-induced movements.

\section{CONCLUSIONS}

It is thus feasible to deliver complex vibration patterns that can generate gait-like movements when applied on a nondisabled participant or a participant with spinal cord injury. The present results warrant evaluating the potential of these complex patterns of vibration for gait rehabilitation in larger pathological groups. 


\section{ACKNOWLEDGMENTS}

\section{Author Contributions:}

Study concept and design: C. Duclos, C. Kemlin, D. Gagnon, J-O. Dyer, R. Forget.

Acquisition of data: C. Duclos, C. Kemlin, D. Lazert.

Analysis and interpretation of data: C. Duclos, C. Kemlin, D. Lazert. Drafting of manuscript: C. Duclos.

Critical revision of manuscript for important intellectual content:

C. Duclos, C. Kemlin, D. Lazert, D. Gagnon, J-O. Dyer R. Forget.

Statistical analysis: C. Duclos, C. Kemlin, D. Lazert.

Obtained funding: C. Duclos, D. Gagnon, J-O. Dyer, R. Forget.

Financial Disclosures: The authors have declared that no competing interests exist.

Funding/Support: This material was based on work supported in part by the Canadian Foundation for Innovation $\left(n^{\circ} 18218\right)$ and the Quebec Rehabilitation Research Network. CD and DG are collaborators of the Canadian Institute of Health Research Sensorimotor Rehabilitation Research Team (http://www.errsm.ca/).

Institutional Review: The research ethics board of the Centre for Interdisciplinary Research in Rehabilitation institutions reviewed and approved this study.

Participant Follow-Up: The authors do not plan to inform participants of the publication of this article.

\section{REFERENCES}

1. Fallon JB, Macefield VG. Vibration sensitivity of human muscle spindles and Golgi tendon organs. Muscle Nerve. 2007;36(1):21-29. [PMID:17471568] http://dx.doi.org/10.1002/mus.20796

2. Roll JP, Vedel JP. Kinaesthetic role of muscle afferents in man, studied by tendon vibration and microneurography. Exp Brain Res. 1982;47(2):177-90. [PMID:6214420] http://dx.doi.org/10.1007/BF00239377

3. Roll JP, Vedel JP, Ribot E. Alteration of proprioceptive messages induced by tendon vibration in man: A microneurographic study. Exp Brain Res. 1989;76(1):213-22.

[PMID:2753103] http://dx.doi.org/10.1007/BF00253639

4. Goodwin GM, McCloskey DI, Matthews PB. The contribution of muscle afferents to kinaesthesia shown by vibration induced illusions of movement and by the effects of paralysing joint afferents. Brain. 1972;95(4):705-48.

[PMID:4265060] http://dx.doi.org/10.1093/brain/95.4.705

5. Hagbarth KE, Eklund G. Tonic vibration reflexes (TVR) in spasticity. Brain Res. 1966;2(2):201-3. [PMID:5968925] http://dx.doi.org/10.1016/0006-8993(66)90029-1

6. Latash ML, Gurfinkel VS. Tonic vibration reflex and position of the body. Fiziologiya Cheloveka. 1976;2(4):593-98.

7. Calvin-Figuière S, Romaiguère P, Gilhodes JC, Roll JP. Antagonist motor responses correlate with kinesthetic illu- sions induced by tendon vibration. Exp Brain Res. 1999; 124(3):342-50. [PMID:9989440]

http://dx.doi.org/10.1007/s002210050631

8. Eklund G, Hagbarth KE. Normal variability of tonic vibration reflexes in man. Exp Neurol. 1966;16(1):80-92.

[PMID:5923486]

http://dx.doi.org/10.1016/0014-4886(66)90088-4

9. Polónyová A, Hlavacka F. Human postural responses to different frequency vibrations of lower leg muscles. Physiol Res. 2001;50(4):405-10. [PMID:11551147]

10. Thyrion C, Roll JP. Predicting any arm movement feedback to induce three-dimensional illusory movements in humans. J Neurophysiol. 2010;104(2):949-59. [PMID:20538782] http://dx.doi.org/10.1152/jn.00025.2010

11. Winter DA. The biomechanics and motor control of human gait: Normal, elderly and pathological. Waterloo (Canada): University of Waterloo Press; 1991.

12. Winter DA. Biomechanics and motor control of human movement. 2nd ed. New York (NY): Wiley; 1990.

13. Wu G, Siegler S, Allard P, Kirtley C, Leardini A, Rosenbaum D, Whittle M, D’Lima DD, Cristofolini L, Witte H, Schmid O, Stokes I; Standardization and Terminology Committee of the International Society of Biomechanics; International Society of Biomechanics. ISB recommendation on definitions of joint coordinate system of various joints for the reporting of human joint motion-part I: ankle, hip, and spine. J Biomech. 2002;35(4):543-48.

[PMID:11934426] http://dx.doi.org/10.1016/S0021-9290(01)00222-6

14. Nelson-Wong E, Howarth S, Winter DA, Callaghan JP. Application of autocorrelation and cross-correlation analyses in human movement and rehabilitation research. J Orthop Sports Phys Ther. 2009;39(4):287-95. [PMID:19346626] http://dx.doi.org/10.2519/jospt.2009.2969

15. Cordo P, Lutsep H, Cordo L, Wright WG, Cacciatore T, Skoss R. Assisted movement with enhanced sensation (AMES): Coupling motor and sensory to remediate motor deficits in chronic stroke patients. Neurorehabil Neural Repair. 2009;23(1):67-77. [PMID:18645190] http://dx.doi.org/10.1177/1545968308317437

16. Gurfinkel VS, Levik YS, Kazennikov OV, Selionov VA. Locomotor-like movements evoked by leg muscle vibration in humans. Eur J Neurosci. 1998;10(5):1608-12. [PMID:9751133] http://dx.doi.org/10.1046/j.1460-9568.1998.00179.x

17. Selionov VA, Ivanenko YP, Solopova IA, Gurfinkel VS. Tonic central and sensory stimuli facilitate involuntary airstepping in humans. J Neurophysiol. 2009;101(6):2847-58. [PMID:19339461] http://dx.doi.org/10.1152/jn.90895.2008

18. Field-Fote E, Ness LL, Ionno M. Vibration elicits involuntary, step-like behavior in individuals with spinal cord 
injury. Neurorehabil Neural Repair. 2012;26(7):861-69. [PMID:22328683]

http://dx.doi.org/10.1177/1545968311433603

19. Maletsky LP, Sun J, Morton NA. Accuracy of an optical active-marker system to track the relative motion of rigid bodies. J Biomech. 2007;40(3):682-85. [PMID:16580000] http://dx.doi.org/10.1016/j.jbiomech.2006.01.017

Submitted for publication April 2, 2013. Accepted in revised form August 28, 2013.
This article and any supplementary material should be cited as follows:

Duclos C, Kemlin C, Lazert D, Gagnon D, Dyer JO, Forget R. Complex muscle vibration patterns to induce gaitlike lower-limb movements: Proof of concept. J Rehabil Res Dev. 2014;51(2):245-52.

http://dx.doi.org/10.1682/JRRD.2013.04.0079

ResearcherID/ORCID: Cyril Duclos, PhD: C-4060-2014 
